\title{
Influence of the Environment on Self-Interaction of Quantum Dot
}

\author{
R.Kh. Gainutdinov ${ }^{a}$, M.A. Khamadeev, M.R. Mohebbifar, and A.A Mutygullina \\ Kazan Federal University, Institute of Physics, 18 Kremlevskaya Str. Kazan 420008, Russia
}

\begin{abstract}
The self-interaction of a quantum dot tunnel with Coulomb interaction coupled to two leads is investigated. We show that actually in the case of quantum dots the variations of the self-energy functions can be strong, and this can have a significant effect on the values of the tunneling-induced shifts of energy levels of quantum dots, that cannot be obtained in the standard way.
\end{abstract}

Quantum dots are nanometer-sized three-dimensional structures which confine electrons in all three directions. This confinement gives rise to a discrete energy spectrum. Quantum dots are of great interest both from a technological and a fundamental point of view. For example, experimental observations of resonance fluorescence from quantum dots showed an essential discrepancy with the predictions of quantum optics for two-level atomic systems [1]. This disagreement can originate from the fact that in the standard theory of resonance fluorescence from a two-level atom the non-radiative transitions caused by the self-interaction processes are not taken into account. In the case of quantum dots the role of the virtual photons in the self-interaction of ordinary atoms can be played by electrons which can leave the quantum dot and then come back. Such a self -interaction can be much more significant than the interaction of an atom with its own radiation field. In this work we investigate the effect of such a self-interaction on the tunneling-induced shift of energy levels of a quantum dot.

Using the generalized dynamical equation the self-energy functions for the states of the quantum dot with one, two electrons and for the state without electrons were derived [2]. With these functions the correction to the energies of the quantum dot that were derived previously [3] in the standard way were calculated. These corrections strongly depend on the variation of the self-energy functions for the corresponding states. If the variations of the self-energy functions were weak enough, then one could restrict oneself to this approximate solution. However [2], this is not the case, and this yields the significant shifts. Thus, by choosing parameters of a quantum dot coupled to leads, it is possible to intensify the self-interaction processes that open the way to increase the light-matter interaction.

\section{References}

1. A.N. Vamivakas et al, Nature Phys 327. P. 840 (2009)

2. R Kh Gainutdinov et al, J. Phys.: Conf. Ser. 613. P. 012002 (2015)

3. J. Splettstoesser, M. Governale and J. König, Phys. Rev. B. 86 P. 035432 (2012)

\footnotetext{
${ }^{a}$ Corresponding author: Renat.Gainutdinov@kpfu.ru
} 